\title{
An Investigation Into the Knowledge of South African Pharmacists on the Identification and Management of Drug-Drug Interactions
}

\author{
Mohammed A. Baksh ${ }^{1}$, Velisha A. Perumal-Pillay ${ }^{1} \&$ Frasia Oosthuizen ${ }^{1}$ \\ ${ }^{1}$ Discipline of Pharmaceutical Sciences, School of Health Sciences, University of KwaZulu-Natal, Durban, South \\ Africa \\ Correspondence: F. Oosthuizen, Discipline of Pharmaceutical Sciences, School of Health Sciences, University of \\ KwaZulu-Natal, Durban, South Africa. Tel: 27-31-260-7242. E-mail: oosthuizenf@ukzn.ac.za
}

Received: August 2, 2019 Accepted: October 10, 2019 Online Published: October 15, 2019

doi:10.5539/gjhs.v11n12p146 URL: https://doi.org/10.5539/gjhs.v11n12p146

\begin{abstract}
Background: Detecting and reporting drug-drug interactions (DDIs) is an important role of pharmacists. Standard operating procedures (SOPs), that can be used to manage DDIs is not a requirement at pharmacies in South Africa. SOPs create standardized methods of identifying and reporting DDIs.

Aim: The aim of this study was to investigate the knowledge of South African pharmacists on the identification and management of DDIs as well as the availability and use of SOPs in the detection and management of DDIs.

Methods: A quantitative approach was used targeting registered pharmacists from two provinces in South Africa, namely Gauteng and KwaZulu-Natal. 153 responses were received after mailing the questionnaire to 200 pharmacists (76.5\% response rate). Data was analysed by using $\operatorname{Microsoft} \operatorname{Excel}^{\circledR}$ and $\operatorname{SPSS}^{\circledR}$ (version 23.0).
\end{abstract}

Results: The majority (93.5\%) of respondents were able to correctly define. Forty-four percent of respondents were aware of the existence of SOPs in their respective pharmacies. The majority of the respondents $(80.4 \%)$ were of the opinion that having SOPs in place for the management of DDIs benefit the identification of these in the pharmacy environment. The findings indicated that availability and access of SOPs are the same across all sectors of pharmacy.

Conclusion: The results show that the majority of participants have a sound knowledge regarding DDIs as well as the importance of reporting them should such events occur. While most pharmacists were not aware of SOPs in their pharmacies, they regarded this as beneficial.

Keywords: drug-drug interactions, adverse drug reactions, standard operating procedures, South Africa

\section{Introduction}

DDIs occur when two or more drugs react with each other resulting in the modification of one or more of the concurrently administered drugs, thereby decreasing their efficacy, resulting in unwanted or toxic effects, and ultimately complete treatment failure (Chatsisvili et al., 2010). DDIs can be divided into three groups depending on the mechanism of the interaction (Admassie, Melese, Mequanent, Hailu, \& Srikanth, 2013). These include pharmacodynamic, pharmacokinetic and pharmaceutical interactions. A pharmacodynamic interaction occurs when two drugs with similar mechanisms of action are concurrently administered resulting in a synergistic or antagonistic effect. A pharmacokinetic interaction occurs when one drug alters the absorption, distribution, metabolism and excretion of another medication. Finally, a pharmaceutical interaction occurs when mixing chemically incompatible drugs outside the body resulting in the inactivation of one or both drugs (Admassie et al., 2013). DDIs can play a significant role in the health outcomes of patients and limiting these DDIs would ultimately benefit patient therapeutic outcomes.

A medicine has many effects. Usually only one effect, being the therapeutic effect, is required for the treatment of a disease. The other effects, whether intrinsically harmful or not, may be termed 'unwanted effects'. These unwanted effects are also referred to as adverse drug reactions (ADRs). ADRs are effects that are unwanted, noxious, unpleasant or potentially harmful (Marsh, 2018). It is the responsibility of healthcare professionals to identify and prevent ADRs which will ultimately result in an improvement in clinical effectiveness of medication. ADRs are one of the important complications of medication therapy that affects more than a million patients globally each year and can have life-threatening consequences (Makiani, Nasiripour, Hosseini, \& Mahbubi, 2017). 
Pharmacists should have a sound knowledge of DDIs and ADRs to improve therapeutic outcomes, clinical effectiveness of medication and reduced hospitalisations. The World Health Organisation (WHO) advises that institutions should have proper systems in place for the early detection of DDIs and medication errors as this could potentially save lives and money (WHO, 2018a). Systems involving the development, training and use of SOPs as a tool are an example that can assist pharmacists to carry out activities to prevent DDIs and ADRs from occurring. In South Africa, SOPs are made available by the National Department of Health and can be accessed online. Having SOPs available in pharmacies can ensure that staff are knowledgeable regarding correct procedures to follow to identify and manage DDIs. SOPs should ideally be standardised across institutions and pharmacies and should include processes and reporting systems that must be adhered to if a DDI is identified.

The standardisation of SOPs can ensure that DDIs are detected and managed the same way across all health care institutions that involve the prescribing and dispensing of medication. Furthermore, SOPs should be updated regularly in line with information such as safety alerts and warnings that are made available by relevant authorities (namely the South African Health Products Regulatory Authority, adverse event reporting units within pharmaceutical companies, and adverse events monitoring and reporting guidelines made available by the National Department of Health with regards to medicines being used and the safety thereof).

This study therefore aimed to determine the knowledge of South African pharmacists in the identification and management of DDIs in two provinces of South Africa namely Gauteng and KwaZulu-Natal (KZN), and to determine the availability and accessibility of SOPs relating to the identification and management of DDIs to practising pharmacists.

\section{Method}

\subsection{Study Sample}

Inclusion criteria were pharmacists working in either private or public sector pharmacies (inclusive of hospital pharmacies) from the provinces KZN and Gauteng. A database containing a list of 928 pharmacists made accessible by the South African Pharmacy Council (SAPC) was identified as the target population for the two provinces. A total of 200 respondents were selected via random sampling. 153 questionnaires were returned which yielded a response rate of $76.5 \%$. Full ethical approval for the study was obtained from the University of KwaZulu-Natal's Biomedical Research Ethics Committee (BE523/17).

\subsection{Sampling, Data Collection and Analysis}

A non-probability sampling technique was used to randomly select a small sample of 200 respondents due to time limitations and onerous of sending out the samples. The software, Microsoft Random Sample Software ${ }^{\circledR}$, was used in the random selection of the sample ensuring that all individuals in the target population had an equal chance of being selected. The questionnaires developed for this study were sent via an online survey cloud-based software, Google forms ${ }^{\circledR}$, in March, 2018 to the selected sample. Feedback from 153 pharmacists was received during the period of March - May 2018 indicating a response rate of $76.5 \%$. On receipt of the electronically completed questionnaires, the relevant data and variables such as age bracket, sex, for each answered question on the questionnaire were transferred to a password protected Microsoft Excel ${ }^{\circledR}$ spreadsheet. No personal details of the respondents were requested.

Descriptive and inferential analyses were conducted on the results of this study. Descriptive analysis included information on frequencies, percentages, measures of central tendency (for example mean, median and mode), and measures of variability (for example range, standard deviation and variance). Inferential analysis was carried out to determine the strength of the relationships (Pearson Chi Square test) between different variables. A difference between variables with a p-value of less than 0.05 was considered to be statistically significant.

\section{Results}

\subsection{Demographic Overview}

One hundred and fifty-three completed questionnaires were analysed of which $66 \%$ of the respondents $(n=101)$ were female and $34 \%(n=52)$ of the respondents were male. The majority of respondents were in the age group of $30-39$ years $(n=63,41.2 \%)$, followed by the age group of 20-29 $(n=29,19 \%), 40-49(n=27,17.6 \%)$ and $>60$ years $(n=24,15.7 \%)$. The age group of 50-59 $(n=10,6.5 \%)$ accounted for the least number of respondents. The mean age was 36 years (standard deviation was 1.3 ).

The majority of the respondents $(58.2 \%)$ were pharmacists $(n=89)$, followed by $24.8 \%$ pharmacy managers $(n=$ $38)$ and $17 \%$ pharmacy supervisors $(n=26)$. The majority of the respondents $(69.3 \%)$ worked in urban areas $(\mathrm{n}=$ $106)$, with only $25.5 \%(n=39)$ from rural areas. 
Most respondents $(62.7 \%)$ work in the private pharmacy sector $(\mathrm{n}=96)$. Forty-six respondents $(30.1 \%)$ worked in public sector pharmacies and $5.2 \%(\mathrm{n}=8)$ in other sectors such as academic institutions, consultancy and non-governmental organisations. Only $2 \%$ respondents work in a private-government partnership $(n=3)$.

The highest number of responses $(53.6 \%)$ were received from pharmacists working in the Gauteng province $(\mathrm{n}=$ $82)$, followed by $41.2 \%$ from KZN $(n=63)$. Eight responses came from pharmacists in provinces other than Gauteng or KZN (5.2\%) - these pharmacists probably moved to another province in the time lapse between the receipt of the name list and the study being conducted .It is important to note that at the time the questionnaires were sent out, they were sent out based on the information contained on the data base made available by the SAPC. This meant that although they no longer worked in KZN and Gauteng, their current registrations with the SAPC still showed that they are registered pharmacists working in either KZN or Gauteng and therefore met the inclusion criteria.

The largest number of respondents worked in public sector hospitals $(n=34,22.2 \%)$. This was followed by pharmacists working in sectors indicated as "other" on the questionnaire $(\mathrm{n}=32,20.9 \%)$. Pharmacists working in private pharmacies accounted for $16.3 \%(\mathrm{n}=25)$, pharmacists working in private sector hospital pharmacies for $15.7 \%(\mathrm{n}=24)$, and pharmacists working in community pharmacies for $13.7 \%(\mathrm{n}=21)$. The least number of responses came from pharmacists working in community health centres $(\mathrm{n}=17,11.1 \%)$.

\subsection{Pharmacists' Knowledge on DDIs and ADRs}

Most respondents were familiar with the term pharmacovigilance $(n=143,93.5 \%)$ and the same number of respondents were able to choose the correct definition of a DDI $(n=143,93.5 \%)$. The number of respondents that were able to choose the correct definition of an ADR was similarly high $(\mathrm{n}=139,90.8 \%)$. The majority of respondents were aware that some DDIs and ADRs could be fatal $(\mathrm{n}=148,96.7 \%)$. The majority of respondents correctly identified contributors to DDIs $(\mathrm{n}=98,64.1 \%)$ and ADRs $(\mathrm{n}=109,71.2 \%)$.

\subsection{Availability of SOPs in Pharmacies}

The findings indicate that approximately the same number of pharmacists were aware of SOP's for the detection and management of DDI's and ADRs in their work place $(n=68,44.4 \%)$ as compared to those who were not aware of any $\operatorname{SOPs}(n=64,41.8 \%)$. A small number of pharmacists $13.1 \%(n=20)$, indicated that they were unsure of the existence of SOPs. More pharmacists $(n=42,27.5 \%)$ stated that SOPs were easily accessible to all dispensing staff at their institution as compared to pharmacists who felt differently $(\mathrm{n}=29,19.0 \%)$. Although accessible, a concerning number of pharmacists $(n=59,38.6 \%)$ stated that staff were not trained on the application of existing procedures.

A large number of respondents $53.6 \%(n=82)$ were either unaware of SOPs or chose not to respond. Of the $44.7 \%$ $(\mathrm{n}=68)$ pharmacists who responded that SOPs do exist in their place of employment for the detection of DDIs, more than half $51.5 \%(n=35)$ stated that these SOPs were not regularly reviewed.

Thirty-two percent of the respondents $(n=49)$ stated that the availability of SOPs helped them in the management of DDIs; the rest of the respondents were not aware of SOPs or chose not to respond to the question. The majority $(80.4 \%)$ of the respondents $(n=123)$ were of the opinion that having SOPs in place benefit the identification of DDIs in the pharmacy as well as the identification and management of ADR $(85.6 \% ; n=131)$.

The availability of $(\mathrm{P}=0.549)$ and access to $(\mathrm{P}=0.746)$ SOPs was the same across all sectors of pharmacy.

\subsection{Recommendations for the Improvement of Managing DDIs in Pharmacies}

Thirty-four percent of the respondents recommended that all dispensing staff within the pharmacy should engage in training programmes in order to create an awareness with regard to the SOPs $(n=52)$. Increasing staff knowledge on DDIs and ADRs (40,3\%), updating SOPs $(34,22.2 \%)$ as well as increasing staff knowledge on reporting systems within existing institutional policies $(40,3 \%)$ were further recommendations for improving the management of DDIs. The use of a computer generated DDI software checker as well as the use of up to date DDI reference material $(n=34,22 \%)$ was also recommended by the respondents.

\section{Discussion}

For over 40 years South Africa has been engaged in pharmacovigilance activities (Mehta et al., 2017). The WHO has defined pharmacovigilance as the science and activities relating to the detection, assessment, understanding and prevention of adverse effects or any other drug-related problem (WHO, 2018b). Pharmacists play pivotal roles in the identification, detection, prevention, and management of DDIs, drug-food interactions and ADRs (Bushra, Baloch, Jabeen, Bano, \& Aslam, 2015). 
The majority of the pharmacists in this study were aware of the concept of pharmacovigilance and the fatalities involved with DDI and ADRs. It is therefore evident that the respondents in this study had an adequate knowledge regarding DDIs and ADRs and knew the importance of DDI and ADR detection and management by pharmacists. Although Pharmacists' knowledge of terminology on DDIs and ADRs was shown to be adequate, their knowledge on correct identification of contributors to DDIs and ADRs fell slightly short, leaving room for improvement and could benefit from structured training programs to guide them in carrying out their pharmacovigilance activities. A study carried out in the North West Province of South Africa found that pharmacists are familiar with the concept of pharmacovigilance, however, less than half reported their familiarity with ADRs (Joubert \& Naidoo, 2016). A study conducted in Kuwait found that more than half of the respondents were knowledgeable about the concepts of pharmacovigilance however, less than half had previously reported an ADR (Alsaleh, Alzaid, Abahussain, Bayoud, \& Lemay, 2017).

In contrast to the results found in this study and the above two studies, a study conducted in India suggested that pharmacists have inadequate knowledge of DDIs and ADRs. It was also suggested these pharmacist should undergo periodic training to aid them in carrying out pharmacovigilance activities, a recommendation that was also highlighted in this study (Rao, Radhakrishnan, \& Andrade, 2011). Similar results to the study conducted in India were found in a study conducted in Jordan whereby pharmacists in Jordan have insufficient awareness and lack of knowledge about pharmacovigilance and ADR reporting (Suyagh, Farah, \& Abu Farha, 2015).

From the above it is evident that pharmacists' knowledge differ; this problem can possibly stem from inadequate education at a tertiary level before even entering the work environment (Saverno, Malone, \& Kurowsky, 2009) or a lack of continuing education.

Pharmacists in this study understood that SOPs can assist them in the era of large patient volumes and new medication constantly being made available on the market; SOPs can guide their practice with up to date information regarding the responsible use and management of DDIs and ADRs. SOPs in a health care setting can accelerate the initiation of therapy for individual patients by increasing the awareness of the need to vigorously and rapidly treat such patients (Kortgen, Niederprüm, \& Bauer, 2006). The Health Outcomes Research Unit and the Evaluation Unit of the KZN Department of Health has prepared guidelines which is directed at creating a standardized methodology of determining the nature, incidence and causes and reporting of adverse health events at hospitals in KZN as well as the design of SOPs for preventative actions (Mahomed, 2006). Regular and consistent training and education programmes on SOPs for pharmacists to detect and manage DDIs will assist them to identify and manage DDIs.

Nearly equal numbers of respondents indicated the availability or unavailability of SOPs in their respective pharmacies. SOPs are "a specific set of practices that are required to be initiated and followed when specific circumstances arise" (Patel, Churi, Gurumurthy, \& Madhan, 2016). SOPs describe the procedures or a set of procedures that can be used as a response to a given event (Rao et al., 2011). If SOPs are introduced at an institution it is important that staff are made aware of this. In a study conducted at a hospital in India, the importance of SOPs was highlighted when the quality of ADR reporting and monitoring improved after the implementation of SOPs (Patel et al., 2016).

The majority of the respondents indicated that although the SOPs assisted them to a great extent, many were not aware or trained to use SOPs. SOPs can provide guidance not only with identifying DDIs and ADRs, but also with proper management including reporting of these. In a study conducted in Lagos, South West Nigeria, the most important reason highlighted for poor reporting of ADRs amongst community pharmacists was the fact that there was a lack of knowledge on how to report an ADR (Oreagba, Ogunleye, \& Olayemi, 2011). In this study, it was emphasised that there is an urgent need for educational programmes and training on pharmacovigilance activities and ADR reporting to increase community pharmacists' willingness to practice it.

A further study conducted in New Delhi, India, found that even though doctors had a good knowledge on ADRs, one of the major reasons highlighted for not reporting ADRs was the lack of set procedures of ADR reporting in their organization (Amrita \& Singh, 2011). A suggestion in the New Delhi study to improve ADR reporting was through implementing workshops. By increasing the awareness level of pharmacy staff on SOPs and training staff on the use of available and accessible regularly updated SOPs will ultimately aid them in the detection and management of DDIs to prevent the occurrence of ADRs.

This study was carried out in only two of the nine provinces of South Africa namely KwaZulu-Natal and Gauteng. Due to time constraints only 200 questionnaires were sent out to pharmacists of which 153 responses were received. Therefore, due to the small sample size and the geographic limitation, the results cannot be extrapolated to the entire country. The study only determined whether pharmacists were knowledgeable on the identification 
and management of DDIs and did not evaluate the quality of the SOPs. Furthermore, only a quantitative approach was used to gather data which did not allow for further probing of responses as is the case of a qualitative approach.

This study may assist pharmacovigilance centers to ensure that pharmacies in both healthcare sectors understand the need for creating SOPs in order to detect and manage DDIs. The findings from this study paves the way for pharmacists to revisit the use of SOPs in their work environment and to ensure that SOPs are used in a responsible manner. It is envisaged that the responsible use of SOPs would ultimately decrease the occurrence of DDIs and incidence of ADRs, thereby reducing patient health care costs, ensuring the rational use of medicines and increasing patient safety. The findings of this study will add to the body of academic knowledge and can be used to create an awareness amongst pharmacists on the importance of the availability, accessibility and regular review of SOPs in the work environment for the detection and management of DDIs and ADRs.

Recommendations for further studies are as follows: (i) to implement successful systems that are found in other countries on SOPs which will educate pharmacy staff on the detection and management of DDIs to prevent ADRs; (ii) for a holistic picture of SOPs related to DDIs and ADRs in South Africa, this study can be expanded to include all provinces of South Africa, and over a longer time period with a larger sample evaluated; (iii) studies evaluating the information contained in SOPs will be of value for quality assurance; and (iv) conducting a qualitative study approach to probe pharmacists responses would add value on the successes or failures of SOP implementation.

\section{Conclusion}

Pharmacists in South Africa, irrespective of the sector they work in, have a knowledge of DDIs and ADRs. While pharmacists understand the benefit of having SOPs in place to identify and manage DDIs and ADRs, many pharmacists were unaware of SOPs in their work environment. Therefore, it is recommended that pharmacists should be introduced to the use of SOPs in order for them to become aware of their availability and accessibility within their work environment. The SOPs should be updated regularly and pharmacists must be informed of the regular update via consistent training programmes.

Of the pharmacist who responded to being aware of SOPs in their work environment, more than half stated that the current SOPs in their work environment were not regularly reviewed. This not only highlighted the need for regular review of existing SOPs, but consistent education on SOPs. Regularly reviewed SOPs containing current National guidelines together with established staff education programmes on SOPs would ensure that every staff member are aware of current SOPs and that SOPS will be used responsibly in order to detect and manage DDIs in order to prevent ADRs.

\section{Acknowledgements}

Dr. V.A. Perumal-Pillay is a University of KwaZulu-Natal (UKZN) Developing Research Innovation, Localisation and Leadership in South Africa (DRILL) fellow. DRILL, is a NIH D43 grant (D43TW010131) awarded to UKZN in 2015 to support a research training and induction programme for early career academics. The content is solely the responsibility of the authors and does not necessarily represent the official views of DRILL and the National Institutes of Health.

\section{Competing Interests Statement}

The authors declare that there are no competing or potential conflicts of interest.

\section{References}

Admassie, E., Melese, T., Mequanent, W., Hailu, W., \& Srikanth, B. A. (2013). Extent of poly-pharmacy, occurrence and associated factors of drug-drug interaction and potential adverse drug reactions in Gondar Teaching Referral Hospital, North West Ethiopia. Journal of Advanced Pharmaceutical Technology \& Research, 4(4), 183-189. https://doi.org/10.4103/2231-4040.121412

Alsaleh, F. M., Alzaid, S. W., Abahussain, E. A., Bayoud, T., \& Lemay, J. (2017). Knowledge, attitude and practices of pharmacovigilance and adverse drug reaction reporting among pharmacists working in secondary and tertiary governmental hospitals in Kuwait. Saudi Pharmaceutical Journal: SPJ: The Official Publication Of The Saudi Pharmaceutical Society, 25(6), 830-837. https://doi.org/10.1016/j.jsps.2016.12.004

Amrita, P., \& Singh, S. (2011). Status of spontaneous reporting of adverse drug reaction by physicians in Delhi. Indian J Pharm Pract, 4(2), 29-36.

Bushra, R., Baloch, S. A., Jabeen, A., Bano, N., \& Aslam, N. (2015). Adverse drug reactions: factors and role of pharmacist in their prevention. Journal of Ayub Medical College, Abbottabad: JAMC, 27(3), 702-706. 
Chatsisvili, A., Sapounidis, I., Pavlidou, G., Zoumpouridou, E., Karakousis, V.-A., Spanakis, M., . . Niopas, I. (2010). Potential drug-drug interactions in prescriptions dispensed in community pharmacies in Greece. Pharmacy World \& Science: PWS, 32(2), 187-193. https://doi.org/10.1007/s11096-010-9365-1

Joubert, M. C., \& Naidoo, P. (2016). Knowledge, perceptions and practices of pharmacovigilance amongst community and hospital pharmacists in a selected district of North West Province, South Africa. Health SA Gesondheid, 21(1), 238-244. https://doi.org/10.1016/j.hsag.2016.04.005

Kortgen, A., Niederprüm, P., \& Bauer, M. (2006). Implementation of an evidence-based 'standard operating procedure' and outcome in septic shock. Critical Care Medicine, 34(4), 943-949. https://doi.org/10.1097/01.CCM.0000206112.32673.D4

Mahomed, O. (2006). Adverse events monitoring and reporting guidelines. Retrieved from http://www.kznhealth.gov.za/family/Adverse-events-monitoring-reporting-guidelines.pdf

Makiani, M. J., Nasiripour, S., Hosseini, M., \& Mahbubi, A. (2017). Drug-drug Interactions: The Importance of Medication Reconciliation. Journal of Research In Pharmacy Practice, 6(1), 61-62. https://doi.org/10.4103/2279-042X.200992

Marsh, D. E. S. (2018). Overview of adverse drug reactions. Retrieved from https://www.msdmanuals.com/home/drugs/adverse-drug-reactions/overview-of-adverse-drug-reactions

Mehta, U., Kalk, E., Boulle, A., Nkambule, P., Gouws, J., Rees, H., \& Cohen, K. (2017). Pharmacovigilance: A public health priority for South Africa. South African Health Review, 2017, 125-133.

Oreagba, I. A., Ogunleye, O. J., \& Olayemi, S. O. (2011). The knowledge, perceptions and practice of pharmacovigilance amongst community pharmacists in Lagos state, south west Nigeria. Pharmacoepidemiology and Drug Safety, 20(1), 30-35. https://doi.org/10.1002/pds.2021

Patel, H., Churi, S., Gurumurthy, P., \& Madhan, R. (2016). Quality Assurance of Adverse Drug Reaction Reporting and Monitoring by Clinical Pharmacists in a Tertiary Care Teaching Hospital. Indian Journal of Pharmacy Practice, 9(1), 27. https://doi.org/10.5530/ijopp.9.1.6

Rao, T. S. S., Radhakrishnan, R., \& Andrade, C. (2011). Standard operating procedures for clinical practice. Indian Journal of Psychiatry, 53(1), 1-3. https://doi.org/10.4103/0019-5545.75542

Saverno, K. R., Malone, D. C. M. P. A. E., \& Kurowsky, J. (2009). Pharmacy Students' Ability to Identify Potential Drug-Drug Interactions. American Journal of Pharmaceutical Education, 73(2), 1-8. https://doi.org/10.5688/aj730227

Suyagh, M., Farah, D., \& Abu Farha, R. (2015). Pharmacist's knowledge, practice and attitudes toward pharmacovigilance and adverse drug reactions reporting process. Saudi Pharmaceutical Journal: SPJ: The Official Publication of The Saudi Pharmacentical Society, 23(2), 147-153. https://doi.org/10.1016/j.jsps.2014.07.001

World Health Organization [WHO]. (2018a). Essential medicines and health products information portal.

World Health Organization [WHO]. (2018b). Essential medicines and health products: pharmacovigilance.

\section{Copyrights}

Copyright for this article is retained by the author(s), with first publication rights granted to the journal.

This is an open-access article distributed under the terms and conditions of the Creative Commons Attribution license (http://creativecommons.org/licenses/by/4.0/). 